\title{
Droni e fotogrammetria moderna per il rilievo dei castelli
}

\author{
Drones and modern photogrammetry for castles
}

\begin{abstract}
Antonio Pecci
Dipartimento di Scienze Umane - Università degli studi della Basilicata, Potenza, Italy, antonio.pecci@unibas.it
\end{abstract}

\begin{abstract}
Castles, often built on hills with extremely steep slopes, or on sea cliffs overlooking stretches of water, were difficult to conquer. Construction techniques and geomorphology of the area were a key factor in making castles impregnable to sieges of military troops or bands of pirates or robbers. Today, the same characteristics make them difficult to survey. In fact, there are huge difficulties in surveying fortified structures on the top of hills or on the edge of a precipice. Such geomorphological features sometimes make the survey difficult, time consuming and expensive and unsafe for operators. Today, these problems can be reduced through the use of drones and photogrammetric processing tools which are based on Structure from Motion algorythms and are easy to use. This method allows us to acquire data with geometric resolution in order to map and study masonry characteristics, as well as analyze and monitor decay and crack patterns for restoration purposes. In this paper, we will discuss the potential of drones and modern photogrammetry techniques in architectural surveys and applied to three case studies. These include the castle of Isabella Morra in Valsinni (Basilicata, South Italy), perched on a cliff; the medieval citadel of Uggiano in Ferrandina (Basilicata, South Italy) in an advanced state of degradation and on a plateau with high geological risk; and the San Fernando Fuerte to Bocochita (Cartagena de Indias, Colombia) overlooking the sea.
\end{abstract}

Keywords: Drones, photogrammetry, castle, risk.

\section{Introduzione}

I castelli e altre strutture difensive, come le torri e le cinte murarie, sono stati costruiti, spesso, in luoghi potenzialmente difficili da assediare. Questa caratteristica si rivela, frequentemente, un enorme problema per il rilievo, la documentazione e per il monitoraggio periodico. Grosse difficoltà sono relative anche alle operazioni di restauro o a specifici interventi strutturali, in quanto richiedono costosi e complessi ponteggi edili. L'utilizzo delle piattaforme UAS (Unmanned Aerial System) consente di risolvere diversi problemi legati al rilievo di territori ed edifici, soprattutto in quei contesti che presentano un al- to livello di difficoltà di documentazione. Questi strumenti di rilievo non convenzionale, possiedono delle prestazioni sempre più elevate e dei bassi costi economici. Le fotografie aeree possono essere scattate mediante delle camere montate su tali veicoli aerei. In questo ambito di applicazione vengono utilizzati soprattutto i droni multirotore, dall'elevata versatilità fornita dalla capacità di hovering, i quali permettono di poter ottenere delle immagini dalle prospettive desiderate. Inoltre, grazie allo sviluppo delle tecniche di fotogrammetria è possibile realizzare anche dei modelli 3D finalizzati alla documentazione 
ma anche alla fruizione virtuale. Da oramai diversi anni i droni e le tecniche fotogrammetriche vengono utilizzate frequentemente per il rilievo del patrimonio culturale (Kourtzellis, 2009; Reu, 2013; Hörr, Brunnett, 2013; Forte, et al., 2015; Pecci, et al., 2015; Campana, 2016), e si contano anche alcuni importanti contributi su applicazioni su castelli o fortificazioni (Almagro, Orihuela, 2015; Rodriguez-Navarro, et al., 2015; Verdiani, 2016; Pecci 2016; Faro, et al., 2017; Bercigli, 2017; Centofanti, et al., 2018; Arciuolo, et al., 2018). Il presente lavoro si pone come obiettivo la discussione delle potenzialità derivate dall'utilizzo dei droni e della moderna fotogrammetria applicata ai castelli.

\section{Casi di studio}

Come casi di studio sono stati scelti il Castello di Isabella Morra a Valsinni (Basilicata, Italia), il Castello di Uggiano a Ferrandina (Basilicata, Italia) e il Forte di San Fernando a Bocochita (Cartagena de Indias, Colombia). Tutti presentano delle difficoltà di rilievo dovute alla morfologia dei luoghi, alle intrinseche caratteristiche architettoniche di difesa, o al loro stato attuale di conservazione.

\subsection{Il Castello di Isabella Morra a Valsinni}

Il Castello di Isabella Morra (Fig. 1) (Pedio, 1987; Santoro, 2014; Scavone, et al., 2015; Vitale, 2018) a Valsinni (Basilicata, MT), fu fondato probabilmente intorno all'anno mille, su un $\mathrm{ca}$ strum longobardo. Successivamente fu feudo dei Sanseverino, Vivacqua di Oriolo, Capaccio, Galeota, Morra, e lega la sua fama alla triste storia della poetessa Isabella Morra. Il castello ha un impianto in parte ancora esistente nelle sue strutture portanti e in parte scomparso o situato al di sotto della quota del cortile attuale, come dimostrano alcune descrizioni sei-settecentesche. L'analisi stratigrafica muraria di alcune pareti del cortile ha inoltre messo in evidenza l'esistenza di una fase storica poca conosciuta e relativa al momento in cui il castello divenne una sorta di fortino di difesa dagli attacchi di briganti, nella seconda metà dell'ottocento. La struttura ha una forma planimetrica irregolare ed insiste su un costone roccioso dominante la vallate del fiume Sinni, le cui pendici degradano fino alle prime case dell'abitato di Valsinni. L'impianto si adegua alla morfologia accidentata del sito e si articola su tre livelli con una superficie di oltre $800 \mathrm{~m}^{2}$.
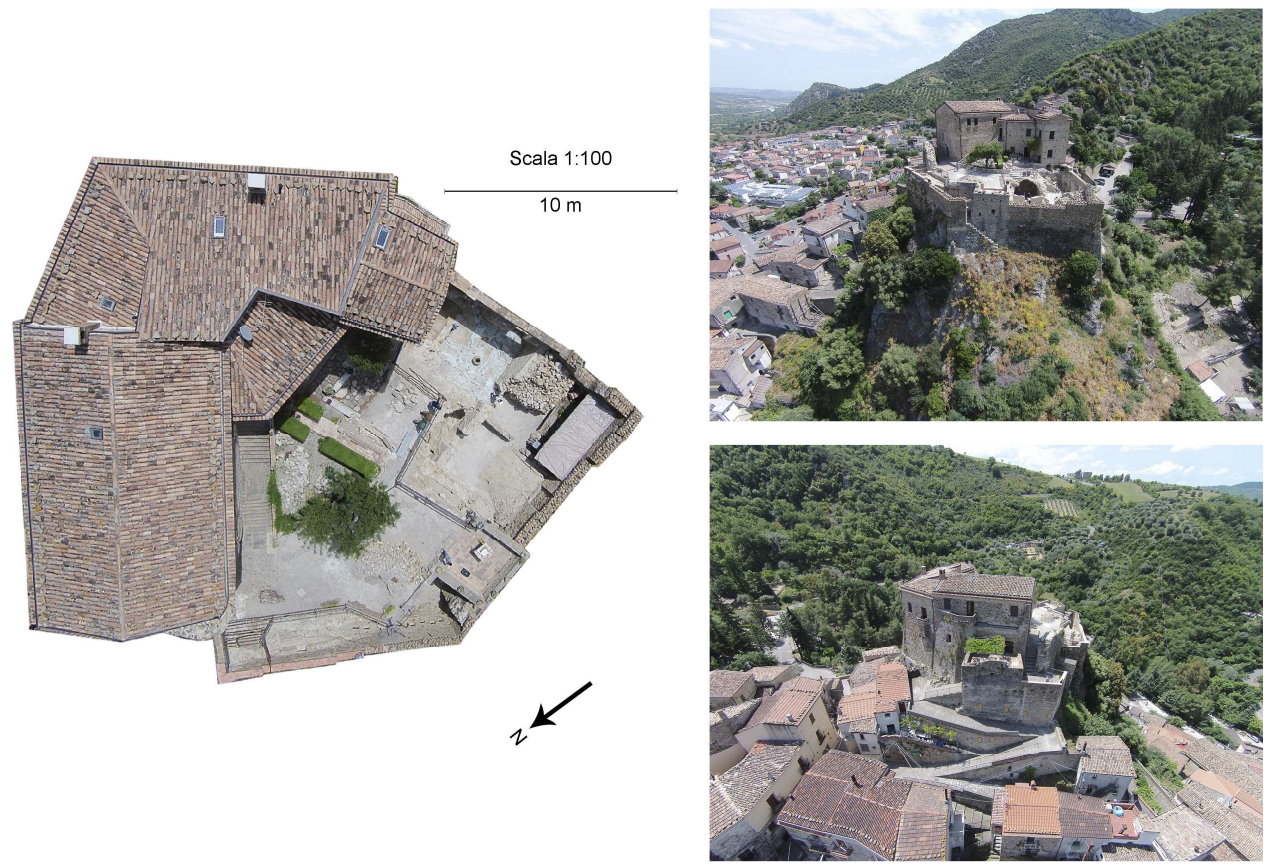

Fig. 1. Castello di Valsinni: planimetria e foto da drone della struttura. 


\subsection{Il Castello di Uggiano}

Il castello di Uggiano (Fig. 2) (Caputi, 1870; Centola 1931; Barbone Pugliese, Lisanti, 1987; Palestina, 1994; Palestina, 2004; Franceschina, 2008; Santoro, 2014, Pecci, 2016; Fiore \& Fumarola, 2018; Pecci, 2019) si trova nel comune di Ferrandina ed è situato sulla sommità di una collinetta le cui pendici degradano verso il canale della Lavannara e la valle della Salandrella. Occupa una superficie di circa un ettaro e il castello segue l'andamento della spianata di sommità. Le prime attestazioni di frequentazione umana della collina del castello risalgono al periodo lucano, ma il primo riferimento cronologico conosciuto risale all'845: Oblano (antico nome del castello), facente parte del Principato di Salerno. Nel 1068 viene conquistato da Roberto il Guiscardo e nel 1275 Uggiano passa a Pietro de Beaumont e a Giovanni Manforte. Successivamente viene acquisito prima da Pirro del Balzo e poi dagli aragonesi. La leggenda vuole che il Castello di Uggiano sia stato abbandonato sul finire del XIV secolo a causa di un sisma e che la popolazione si sia spostata nell'odierna Ferrandina. Circostanza da ritenersi scarsamente probabile per svariati motivi di carattere archeologico e storico. Probabilmente, a giudicare da alcuni evidenti segni di colpi di bombarda visibili sulle murature, a determinare il definitivo abbandono fu un evento bellico. Attualmente sono ancora visibili, in parte, le mura merlate, alcune torri e un arco angioino a sesto acuto. L'intero sito giace in stato di rudere e in un precario stato di conservazione, causato anche da problemi idrogeologici che interessano l'intera collina.

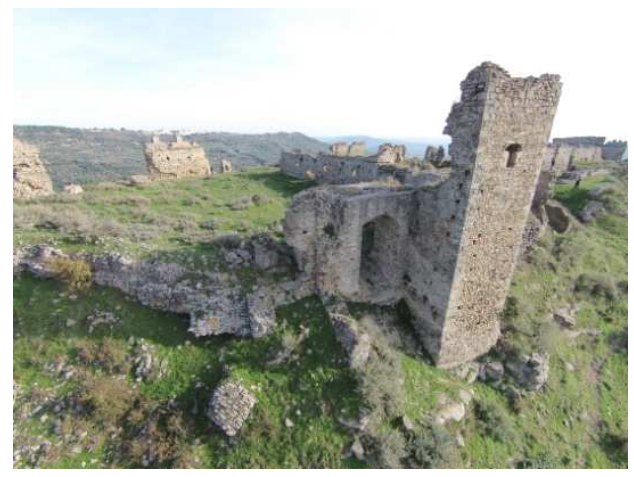

Fig. 2. Ingresso del Castello di Uggiano. Foto da drone.

\subsection{Forte di San Fernando a Bocochita}

Il Forte di San Fernando a Bocochita (Cartagena de las Indias, Colombia) (Gutierrez, Paolini, 1994; Campos, 2003; Paradiso, et al., 2013; Paradiso, et al., 2014) è una delle costruzioni fortificate più importanti dell'antica città coloniale di Cartagena. Situato sull'isola di Tierrabomba, era a difesa dello stretto di Bocochita, l'unico canale navigabile di accesso alla baia della città caraibica di Cartagena e al suo porto. Il forte si data alla seconda metà del XVIII e fu progettato dai più grandi ingegneri militari dell'epoca come Don Ignacio de Sala, Juan Bautista Mac Evan y Antonio de Arévalo. L'intera struttura occupa una superfice di circa $5700 \mathrm{~m}^{2}$ ed è formato da un corpo principale a forma di ferro di cavallo, con la parte curva verso il mare. All'interno di questa struttura è presente un'ampia piazza d'armi e lungo tutto il perimetro si dislocano diversi ambienti funzionali al forte. Alle estremità della struttura fortificata ci sono due baluardi poligonali (el baluarte del Rey e el baluarte de la Reina) posti di fronte alla spiaggia. Il muro perimetrale ha un'altezza cha va dagli 8 ai $10 \mathrm{~m}$. Di fronte al forte si collocano un pendio artificiale che circonda il fossato, e due batterie laterali (la batería de Santiago e la batería de San Juan Francisco Regis). Nel 1958 è stato riconosciuto Patrimonio dell'Umanità dall'UNESCO.

\section{Strumenti e metodo di rilievo}

Il drone utilizzato per il rilievo dei tre casi di studio è il Phantom Vision 2 + della DJI, multinazionale cinese. Si tratta di un quadricottero, dal peso di 1242 grammi, con 4 motori brushless dotato di bussola, altimetro, GPS, con un'autonomia di circa 20 minuti. Fornito di una fotocamera di serie da 14 Megapixel, il drone è pilotato con un radiocomando a 5,8 GHZ. Tramite un mobile device con sistema operativo android o iOS, dotato dell'APP dedicata DJI VISION, è possibile impostare la videocamera e la fotocamera, cambiare i parametri, scegliere la modalità di ripresa (foto/video), la modalità di scatto, controllare la telemetria durante il volo, il livello della batteria, orientare il gimbal a due assi e visualizzare ciò che "vede" il drone grazie ad un collegamento WIFI a 2,4 GHZ. 


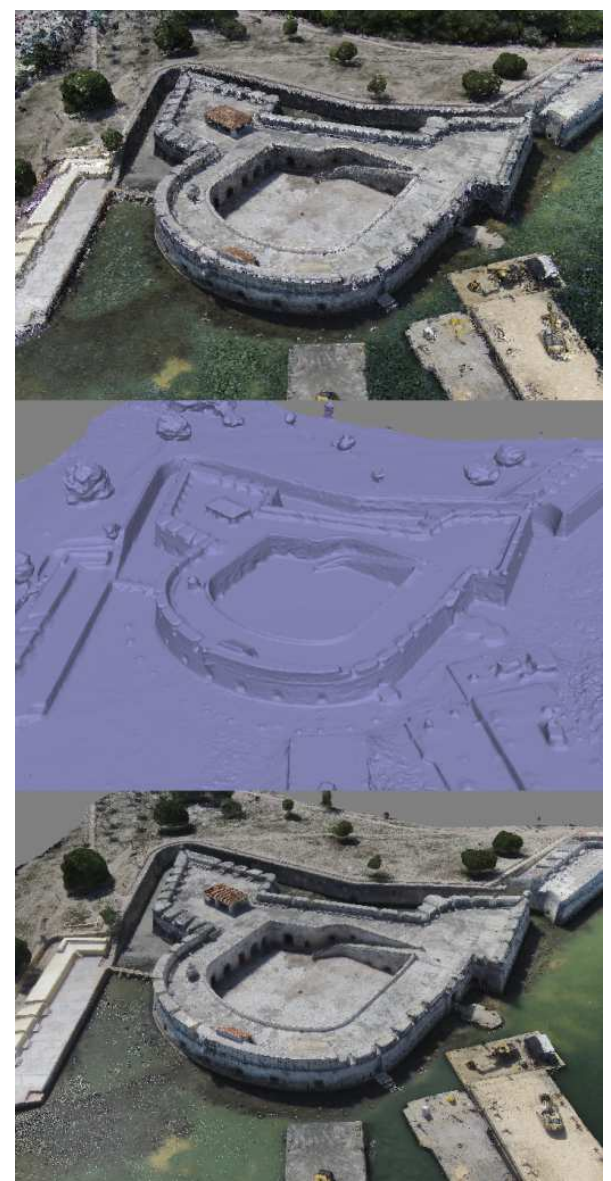

Fig. 3. Workflow di elaborazione del modello 3D del Forte di San Fernando: point cloud, mesh, textured mesh.

In fase di pianificazione della campagna di rilievo si è scelto di effettuare diversi voli, di cui il primo per realizzare una copertura zenitale ed il secondo per quella obliqua riprendendo i castelli a 360 gradi, scattando foto ad intervalli di 3 secondi. Per la ripresa zenitale sono state realizzate due strisciate di acquisizione a quote diverse: la prima a $35 \mathrm{~m}$ e la seconda a $50 \mathrm{~m}$ circa. Nel caso dell'acquisizione in obliquo si è fatto affidamento all'esperienza del pilota per volare a vista, ad una distanza costante dal monumento, controllando l'inquadratura della fotocamera sullo smartphone. Il volo in manuale ha consentito di poter riprendere alcuni dettagli che risultava difficile acquisire con il volo in automatico o pericoloso per via di alcuni potenziali ostacoli come i cavi dell'elettricità, strutture circostanti, alberi, etc. In questo modo è stato possibile acquisire le foto da prospettive e altezze diverse, in modo da poter cogliere più dettagli. Tale modalità di acquisizione permette, soprattutto, al software di Structure from Motion di poter creare dei modelli $3 \mathrm{D}$ più precisi rispetto a quelli realizzati con l'utilizzo di sole immagini aeree scattate ad un'altezza e distanza fissa.

Le foto, dopo essere state corrette mediante il plug-in Camera Raw di Adobe Bridge, sono state elaborate attraverso Agisoft Photoscan; al fine di realizzare modelli 3D ad elevato livello di dettaglio e precisione sono stati utilizzati degli alti parametri di processamento, i cui risultati sono riportati di seguito (Tab. 1).

\begin{tabular}{|l|l|l|l|}
\hline 3D Model & $\begin{array}{l}\text { C. di } \\
\text { Isabella } \\
\text { Morra }\end{array}$ & $\begin{array}{l}\text { C. di } \\
\text { Uggiano }\end{array}$ & $\begin{array}{l}\text { F. di San } \\
\text { Fernando }\end{array}$ \\
\hline N. Foto & 633 & 623 & 643 \\
\hline $\begin{array}{l}\text { Point } \\
\text { cloud } \\
\text { (punti) }\end{array}$ & 48234104 & 47456503 & 50464567 \\
\hline $\begin{array}{l}\text { Mesh } \text { (fa- } \\
\text { ces) }\end{array}$ & 45456302 & 43657854 & 47658932 \\
\hline $\begin{array}{l}\text { Precisione } \\
\text { ortofoto } \\
\text { (mm/pix) }\end{array}$ & 4.7 & 4.4 & 4.2 \\
\hline $\begin{array}{l}\text { Precisione } \\
\text { DEM } \\
\text { (cm/pix) }\end{array}$ & 1.43 & 1.32 & 1.16 \\
\hline
\end{tabular}

Tab. 1. Dettagli tecnici dei modelli 3D realizzati.

Questo software è basato su un work-flow semiautomatico che gestisce tutta la fase di lavoro, composta dall' orientamento delle foto, dalla generazione di una nuvola di punti, dalla creazione di una mesh e del modello 3D finale coperto dalla una texture ad altissima risoluzione (Fig. 3). Di fondamentale importanza è la messa in scala del modello 3D, vengono misurati alcuni punti certi (angoli di strutture, pali di recinzione, marker creati appositamente, etc.) attraverso l'utilizzo di una stazione totale o di un GPS a doppia frequenza e riportate queste misure sul modello tridimensionale attraverso software dedicati. Infine, dal modello 3D realizzato e attra- 
verso Agisoft Photoscan, è stato possibile la creazione di output finali come ortofoto, DEM (Digital Elevation Model) e Google KMZ, gestibili all'interno di software GIS e CAD.

\section{Risultati ottenuti}

Le potenzialità e i vantaggi delle piattaforme UAV e delle tecniche di fotogrammetria moderna nel rilievo dei castelli sono state diverse (Fig. 4).

L'utilizzo del drone ha innanzitutto permesso di ridurre i rischi per l'operatore. Infatti, non accedendo direttamente sul sito, si sono fortemente ridotte le possibilità di incorrere in pericoli come improvvise frane, smottamenti, o caduta dei ruderi (castello di Uggiano); legati ad un pendio estremamente ripido (castello di Isabella Morra); o alla presenza del mare (forte di San Fernando). Allo stesso tempo, l'utilizzo del drone, si è rivelato in tutti e tre i casi l'unico metodo speditivo di rilievo da poter adottare, considerando la posizione dei castelli e la morfologia dei luoghi. In questo modo si sono evitate lunghe e complesse acquisizioni da laser scanner, da stazione totale o da rilievo tradizionale, i quali risultavano complicati (se non con dei barconi nel caso del Fuerte o con l'utilizzo di funi e corde per i paramenti esterni di Valsinni), se non impossibili da effettuare. Nel caso del castello di Uggiano il modello 3D è stato fondamentale per lo studio della staticità del sito e per poter effettuare gli studi necessari per un'urgente messa in sicurezza dell'intera collina e delle strutture. Allo stesso tempo, periodicamente, vengono effettuati dei voli per il monitoraggio costante del sito, al fine di prevenire ulteriori ed eventuali crolli strutturali. Per tutti i castelli sono stati realizzati dei $3 D$ model attraverso le metodologie fotogrammetriche. Grazie al loro elevatissimo livello di dettaglio, hanno permesso di realizzare planimetrie, sezioni e prospetti, su cui sono stati sviluppati i quadri fessurativi e le mappe del degrado (Fig. 5).

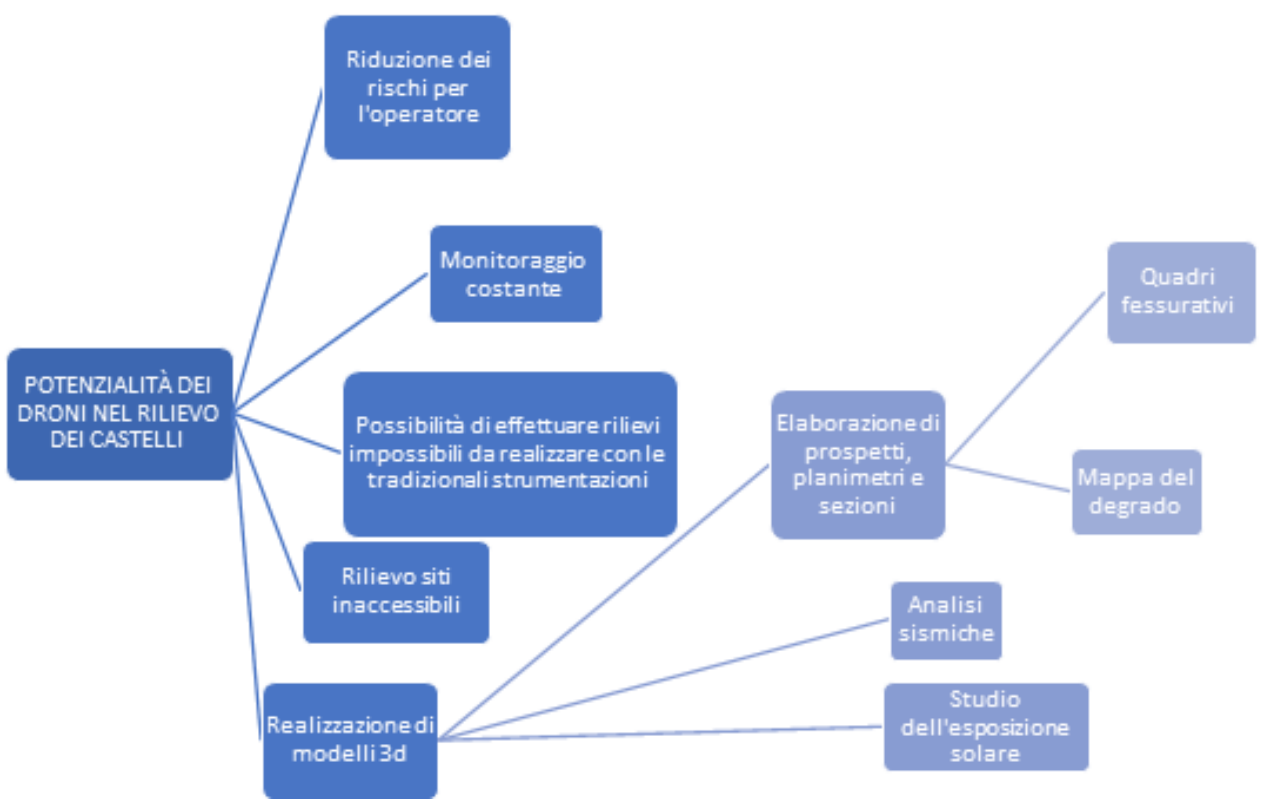

Fig. 4. Potenzialità dei droni nel rilievo dei castelli. 

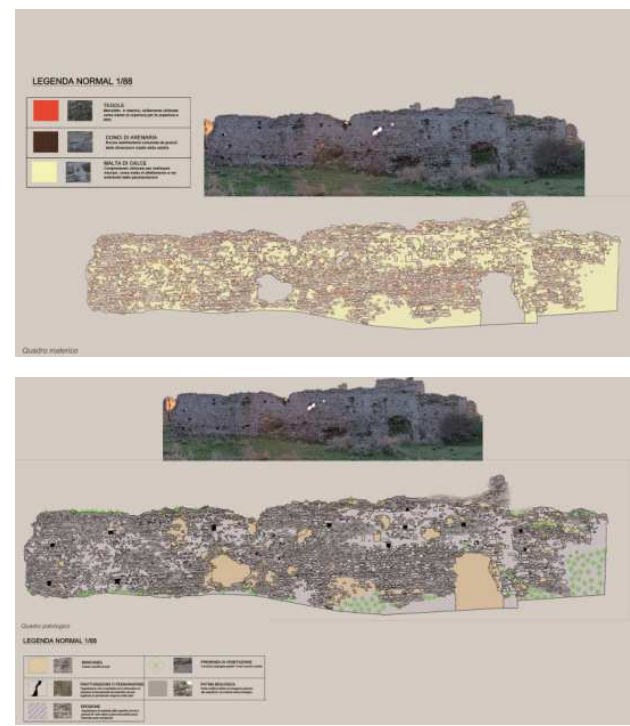

Fig. 5. Quadro fessurativo e mappa del degrado del muro nord della Sala del Castello di Uggiano.

Inoltre, i modelli 3D possono presentare anche un valore aggiunto in alcuni studi ingegneristici come le analisi sismiche o lo studio dell'esposizione solare. Infine, possono essere anche fruiti virtualmente se caricati su apposite piattaforme web come Sketchfab o in apposite APP. È il caso, ad esempio, del castello di Isabella Morra, osservabile on-line in tre dimensioni e all'interno dell'APP per Android Basilicastle (Biscione, et al., 2016).

\section{Conclusioni}

Come si è tentato di dimostrare, i droni e le tecniche di fotogrammetria consentono di realizzare rilievi ad altissimo livello di dettaglio e risoluzione. In determinati casi sono l'unico strumento che permette un rapido rilievo, riducendo i costi derivati e i problemi logistici (voli con elicottero, utilizzo di impalcature, etc.). Ma allo stesso tempo, esistono alcuni limiti come i lunghi tempi di elaborazione dei modelli 3D, la necessaria pianificazione del volo per cercare di ridurre al minimo i problemi di luce e di meteo avverso, una strumentazione di un certo livello e soprattutto un know-how di acquisizione, elaborazione gestione e interpretazione dei dati non indifferente.

\section{Bibliography}

Almagro, A.; Orihuela, A. (2015). "Métodos para documentar y restaurar zonas de difícil acceso en el Castillo de Salobreña. (Granada)", in FORTMED 2015. Defensive architecture of the mediterranean: XV to XVIII centuries, Editorial Universitat Politècnica de València, València, vol. I, pp. 287-290.

Arciuolo, L.; Orangerb, S.; Petruzzelli, R. (2018). "Torre Mattoni, unicum tra le fortificazioni costiere pugliesi. Il progetto di conservazione e di restauro", in Marotta, A.; Spallone, R., eds., FORTMED 2018. Proceedings of the International Conference on Modern Age Fortification of the Mediterranean Coast, vol. VIII, pp. 425-432.

Bercigli, M. (2017). Documentation strategy for coastal towers of the Mediterranean: the case of the tower in the archeological site of Saturo (TA-ITA).

Biscione, M.; Scavone, M.; Danese, M.; Pecci, A.; D'Antonio, A.; Sileo, M.; Masini, N. (2016). "Basili Castle: the digital Atlas of Castles in Basilicata (Southern Italy)", in Defensive Architecture of the Mediterranean XV to XVIII Centuries, vol. IV, pp. 537-544.

Campana, S. (2017). "Drones in Archaeology. State-of-the-art and Future Perspectives", Archaeological Prospection.

Campos, D.P. (2003). Investigatión del Fuerte de San Fernando de Bocachica: una vision integral, Valencia Editorial de la UPV, Valencia.

Caputi, N. (1870). Cenno storico sull'origine progresso e stato attuale della Città di Ferrandina, Napoli

Centofanti, M.; Brusaporci, S.; Lucci, A.; Maiezza, P.; Tata, A. (2018). "Il castello di Pacentro (AQ). Rilievo e conoscenza", in Marotta, A.; Spallone, R., eds., FORTMED 2018. Proceedings of the International Conference on Modern Age Fortification of the Mediterranean Coast, vol. VIII, pp. 511-518.

Centola S. (1931). Ferrandina e le sue remote origini ellenico-lucane, Stab, Tipo-lito Manzoni e De Lucia, Napoli. 
Faro, A. Lo; Rodríguez-Navarro, P.; Santagati, C.; Mangani, M. (2017). Coastal towers in the Mediterranean of XVI century: a comparison between Sicilian and Valencian cases.

Fiore, C.; Fumarola, F. (2018). "Il castello di Uggiano a Ferrandina (MT)", Marotta, A.; Spallone, R., eds., FORTMED 2018. Proceedings of the International Conference on Modern Age Fortification of the Mediterranean Coast, vol. VIII, pp. 627-634.

Forte, M.; Dell' Unto, N.; Jonsson, K.; Lercari, N. (2015). "Interpretation Process at Çatalhöyük using 3D”, in 20th Annual Meeting of the European Association of archaeologists (EAA2014), 10-14 September 2014, Istanbul.

Franceschina, F. La. (2008). "Il Castrum di Uggiano: un abbandono di sei secoli”, in Basilicata Regione Notizie, pp. 119-120.

Gutierrez, R.; Paolini, R. (1994). El Caribe Fortificado, Escala Ediciones, Bogotá.

Hörr, C.; Brunnett, G. (2013). "Boon and Bane of High Resolutions in 3D Cultural Heritage Documentation", in Bock, H.G.; et al., eds., Scientific Computing and Cultural Heritage, Springer-Verlag Berlin Heidelberg, pp. 3139.

Kourtzellis, Y. (2009). "A Critical Approach to Digital Three-Dimensional Representation of Monuments [Kritiki prosegisi ton psifiakon 3D anaparastaseon mnimeion]", Archaiologia kai texnes, 113, pp.11-16.

Lisanti, N.; Barbone Pugliese, N. (1987). Ferrandina recupero di una identità culturale (Catalogo della mostra, Maggio-Luglio 1987), Congedo Editore, Galatina.

Palestina, C. (1994). Ferrandina. Volume quarto. Appendice documentaria, Appia 2 editrice, Venosa.

Palestina, C. (2004). Ferrandina "Uggiano vecchia”, Potenza.

Paradiso, M.; Galassi, S.; Benedetti, S. (2013). "Una contribución para conocer El Fuerte San Fernando De Bocachica, Cartagena De Indias, Colombia", Revista M, 10(2), pp. 136-158.

Paradiso, M.; Galassi, S.; Sinicropi, D. (2014). "Il caratteristico Forte di San Fernando de Bocachica, Colombia", in Villegas, L.; lombillo, I.; Liano, C.; Blanco, H., eds., Patologia della Costruccion, Tecnologia de la Rehabilitacion y Gestion del Patrimonio, Gráficas Iguña, S.A., Proc. Of Congress on Construction Pathology, Rehabilitation Technology and Heritage Management (Rehabend 2014), Santander.

Pecci, A. (2016). "Fonti scritte e UAV per lo studio della topografia del Castello di Uggiano (Ferrandina, Basilicata, Italia)", in FORTMED 2016. Defensive architecture of the mediterranean: XV to XVIII centuries, pp. 45-52.

Pecci, A. (2019). Ferrandina. Guida alla città di Federico D’Aragona, Zaccara Editore, Lagonegro.

Pecci, A.; Scavone, M.; Masini, N.; Sileo, M.; Dantonio, A.; Marzo, C., (2015). "Tecnologie innovative per i Beni Culturali: i Droni”, in Atti del Congreso Internacional sobre Documentación, Conservación y Reutilización del Patrimonio Arquitectónico - La Experiencia del ReUSO. (Valencia, 22-24 Ottobre 2015), pp. 586-593.

Pedio, T. (1987). La Basilicata dalla caduta dell'impero romano agli Angioini, Levante Editori, Bari, vol. III-IV.

Reu, J. De; Plets, G.; Verhoeven, G.; Bats, M.; Cherretté, B., Maeyer, W. De; Smedt, P. De; Deconynck, J.; Herremans, D.; Laloo, P. De; Van Meirvenne, M.; Clercq, W. De. (2013). "Towards a three-dimensional cost-effective registration of the archaeological heritage", Journal of Archaeological Science, 40(2): pp. 1108-1121.

Rodriguez-Navarro, P.; Verdiani, G.; Gil Piqueras, T. (2015). "Comprehensive methodology for Documenting the Defense Towers of the Valencian Coast (Spain)", in FORTMED 2015. Defensive architecture of the mediterranean: XV to XVIII centuries, Editorial Universitat Politècnica de València, València, vol. I, pp. 321-328.

Santoro, L. (2014). Castelli, mura e torri della Basilicata, Canestrini, F., ed., ArtstudioPaparo, Napoli.

Scavone, M.; Masini, N.; Emanuele, F.; Lisanti, L. (2015). "Il progetto di Restauro e Riuso del Patrimonio Architettonico", in III Congreso Internacional sobre Documentación, Conservación, y Reutilización del Patrimonio Arquitectónico, Editorial Universitat Politècnica de València, Valencia.

Verdiani, G. (2017). Fortifications and documentation: the case of Fortezza Vecchia in Livorno. State of the digital survey.

Vitale, V. (2018). "The case of the middle valley of the Sinni (Southern Basilicata). Methods of archaeological and architectural documentation: 3D photomodelling techniques and use of RPAS", Digital Applications in Archaeology and Cultural Heritage, vol. 11. 
